\title{
DESIGNING XML SCHEMA OF A COMPETENCE STRUCTURE FROM COURSE INTENDED LEARNING OUTCOMES
}

\author{
Athitaya Nitchot, Lester Gilbert, Gary B Wills \\ Learning Society Lab, School of Electronics and Computer Science \\ University of Southampton \\ Southampton, SO17 1BJ, United Kingdom \\ $\{$ an08r, lg3, gbw $\} @$ ecs.soton.ac.uk
}

\begin{abstract}
In a traditional classroom the intended learning outcomes have been formed according to the scope of the learning and teaching aims and also based on the structure of course program. It indicates what the learners will learn and the knowledge they will obtain at the end of the course of study. Over the past few years the trend towards a Web-based instructional system has been introduced to the learning and teaching environment. Currently there are many learning materials which are found on the Web. However, this can make it difficult for learners to choose the right study materials which would match with what learners have already learnt and what they would like to know. In this research we consider a learner competence which indicates the differing level of intended learning outcomes depending upon the context of the learners' performance. We propose a competence-based system for recommending study materials from the Web based on the learner's competences. The design of system is considered from a competence structure and we map it into a form of XML schema. By considering the schema, the system can be modifiable and reusable for other knowledge domains. Hence the system not only identifies the learner's competence and suggests study material links from the Web but also the developer can design the competence structure and import it into the system for the future use.
\end{abstract}

\section{KEY WORDS}

"Competency Model" "Competence Structure" "Webbased Education" "Semantic Web"

\section{Introduction}

Currently there are several online materials available on the Web. The benefit of representing study materials on the Web is that learners can access them at any time and from any place and the content of the Web can be easily updated. In order to obtain study materials on the Web, normally learners directly go to a Web page location or use search engines to find the materials that are related to their description (or keywords). Sometimes the learners may realize the difficulties in finding the materials which are matched with what they already known and what they would like to learn. This paper aims to propose a competence-based system for suggesting study material links from the Web to learners and an approach of constructing XML schema of competence structure from a course's intended learning outcomes. For our research, a competence indicates learners' intended learning outcomes based on the different contexts of learner performance. Within the process the system provides lists of competences in a particular knowledge domain for the learner to choose and the chosen competences will be considered as keyword inputs for the search engine (such as Google). The lists provided are generated from the structure of competence elements. The structure identifies the parent-child relationship of competences. It normally indicates what would be the prerequisite competence of one to another competence. The design of the structure is based on a course's intended learning outcomes which have been used for outlining the scope of learning and teaching aims. In this research, we construct the XML schema for a competence structure of a photosynthesis (key stage 4) knowledge domain. The idea of applying XML schema in our system design is to allow for reusability and modification within a competence structure. In addition, the structure of competence can be embedded within the system and used by many learners for learning the same knowledge domain. Hence the required update is considered for only a competence structure not for the learning materials.

The structure of this paper is as follows. Firstly we propose our research approach, competence model, process within a system and a previous developed competence structure. Secondly there is an illustration of the way to design a competence structure. Thirdly the generated class diagram and structure of mapped XML schema have been introduced. In the fourth section the interface of current system will be provided. In the fifth section there are some discussions on the semantic approach and a comparison of XML with other types of structure and database. The paper finally provides a discussion of future works and some conclusions.

\section{Competency Model and System Design}


In this research we propose a system which suggests study material links from the Web to a learner. In this section the research approach and objective will be introduced. There is a discussion of different models of competency and the competency model which is considered within the design of the system.

\subsection{Research Approach}

The aim of this research is to contribute a competencebased system which recommends itself to learners, in order to get appropriate study materials as links from the Web without any communication from the teacher's side. The system is designed not for replacing the teacher role or reducing number of teachers in the classrooms, but the main objective of the system is to help the learners finding study materials as supplementary resources outside of the classrooms. The system aims to suggest study material links corresponding to what the learner would like to learn (desired competence) and what the learner already knows (existing competence). The system process is considered based upon a structure of competence elements in a specific knowledge domain. We aim to design an XML schema of competence structure which is used to define the terms and implicit relationships within a structure. The benefits of this research's system are to identify learners' existing/desired competences and to provide appropriate study materials as links from the Web according to their competences. In addition, this allows a competence structure to be modifiable depending upon different knowledge domains of subject matter content.

\subsection{Competency Model}

The standard definition of competency given in the documentation of competencies (measurable characteristics) by HR-XML consortium [1] is:

"A specific, identifiable, definable, and measurable knowledge, skill, ability and/or other deployment-related characteristic (e.g. attitude, behaviour, physical ability) which a human resource may possess and which is necessary for, or material to, the performance of an activity within a specific business context."

The concept of competence has been dealt with in an educational area [2] and a professional development [3]. In professional development competences are considered as a criterion to select the most appropriate available person for a given task [3]. In the education system, competence could be used to describe final attainment levels of educational programs [2]. There are existing competency standards, for example IMS RDCEO [4] and HR-XML [5]. Their data model is minimalist but extensible for defining competencies or learning objectives. A discussion about these two competency standards is given by Sampson and Fytros [6]. The discussion introduces some drawbacks of these competency standards such as the titles and descriptor elements in these models not being directly machine- understandable. Moreover, both standards adopt competence description but do not take "Proficiency Level" into consideration although a proficiency level is important to the competency concept [6].

The considered model for this research draws on the multi-dimensional competency model (called COMBA) from Sitthisak, Gilbert and Davis [7]. This considers the learners" "learned capability" instead of their "knowledge level". The COMBA model consists of three major components: subject matter, capability, and context (figure 1).

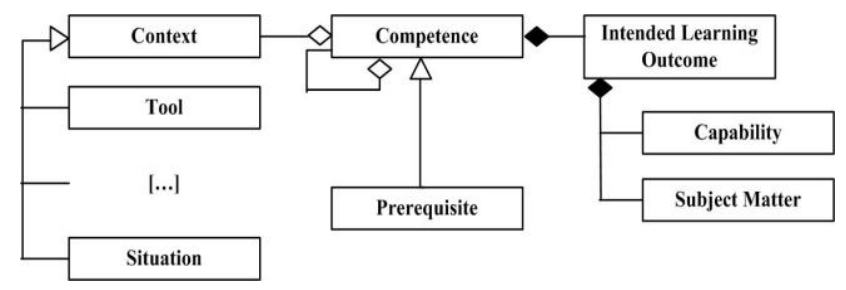

Figure 1. COMBA Competency Model [7]

The considered COMBA competency model incorporates the intended learning outcome which can be formally described as the composition of capability and subject matter. In addition, there are different levels of proficiency in a given intended learning outcome depending upon the context of their performance.

\subsection{System Process}

Figure 2 shows the overview of the process within a system design.

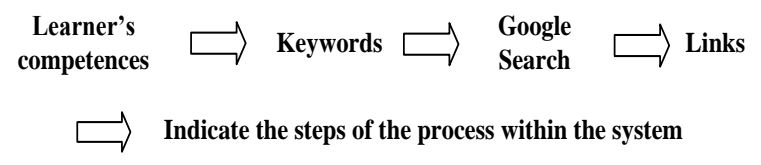

Figure 2. Overview of Process within the System

For our research, there are two kinds of learner's competences: desired and existing competence. Desired competence refers to the learner's intended learning outcome or the competence which the learner wishes to learn. The current or existing competence is the estimation of the actual competence of the learner. The generated key words for Google are considered from corresponding chosen competences. The options for desired and existing competences, which will be chosen by the learner, depend on a structure of competences elements (competence structure).

\subsection{Previously Developed Competence Structure}

A competence structure is considered to specify the range of competence elements/nodes for a particular knowledge domain. The competence structure highlights the relationship between competence nodes and the gap between desired and existing competence. Consideration 
should be given initially to one sample of knowledge. In our first implementation consideration has been given to the knowledge domain: mathematical factor, common factor and highest common factor (H.C.F). An appropriate competence structure for this domain is represented by DAG (directed acyclic graph) as is shown in figure 3 .

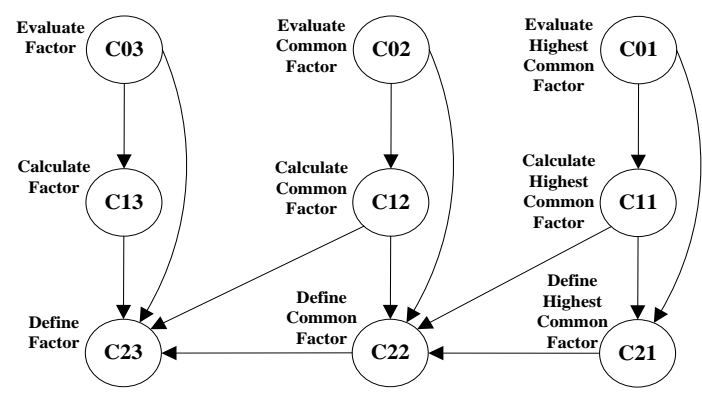

Figure 3. DAG Representation for Competences in H.C.F., Common Factor and Factorisation Domains

The edge between two nodes indicates the parentchild relationship. The arrow heads to the child node which is a prerequisite node. The structure of competence can be conducted by one people. It can be embedded within the system and used by many learners for learning particular knowledge domain. For future use the system can be used for several knowledge domains by only updating the competence structure.

\section{Designing a Competence Structure}

In the previous section the competency model was discussed. The major component of the model is an intended learning outcome. In order to design a competence structure we are required to get information the intended learning outcomes for specific subject matter content of a course. Then we analysis their structure of into a categorization of subject matter content and tag each subject matter content with capability and context in order to get a structure of competence.

\subsection{Intended Learning Outcome}

E-Learning transaction (figure 4) refers to the lowest unit of analysis in learning and teaching [8]. An e-learning transaction is a generalisation from Laurillard's conversational model [9] which is a model that describes the learning and teaching environment in higher education. The heart of an e-learning transaction (figure 4 ) is the purpose (or an intended learning outcome) which is implied from the aim of the overall learning and teaching transaction. The purpose indicates the learner's motivation and it will be the objective of the e-learning development including the use of any learning materials or teaching assets [8].

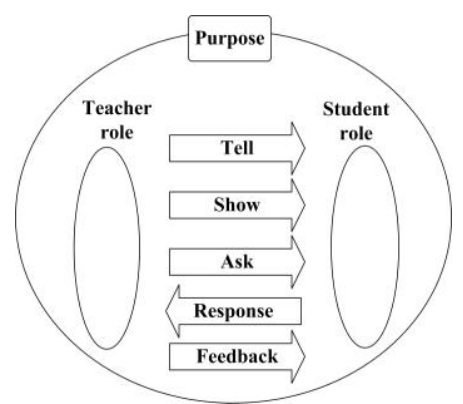

Figure 4. E-learning Transaction [8]

In order to construct a competence structure, we consider the available published intended learning outcomes in UK education or national public syllabus, for example AQA, OCR and Edexcel. For our second implementation the chosen knowledge domain is photosynthesis for a key stage 4 learner. In our research, we consider all the intended learning outcomes of the photosynthesis domain at a key stage 4 (GCSE) from AQA - revised version [10]. Examples of considered intended learning outcomes are as follows:

- recall photosynthesis equation

- recall photosynthesis definition

- define chlorophyll

- interpret data showing how factors affect the rate of photosynthesis

- demonstrate a photosynthesis procedure

- $\quad$ predict the rate of photosynthesis in different condition using computer simulations

\subsection{Constructing a Competence Structure}

From all the intended learning outcomes we summarize them into a list of subject matter as in a table 1 . The first step is to consider the structure of the subject matter content in an e-learning system. This is undertaken by focusing on the broad understanding of the knowledge and cognitive skills of students, in order to achieve the goal. This is called in short 'subject matter content' and is normally categorised into four fields based on Merrill's analysis [11]. They are: fact, concept, procedure and principles.

Table 1

Subject Matter Content according to Intended Learning Outcomes

\begin{tabular}{|l|l|}
\hline $\begin{array}{l}\text { Subject Matter } \\
\text { Type }\end{array}$ & Subject Matter \\
\hline Fact & $\begin{array}{l}\text { Photosynthesis equation, } \\
\text { Photosynthesis definition, Substance, } \\
\text { Energy, Sun, Bulb, Gas, CO2, H2O, } \\
\text { O2, Plant cell, Location, Mesophyll } \\
\text { cell, etc }\end{array}$ \\
\hline Concept & Chlorophyll, Light, Carbon dioxide, \\
& Water, Oxygen, Chloroplast, etc \\
\hline Procedure & Photosynthesis procedure \\
\hline Principle & Photosynthesis rate \\
\hline
\end{tabular}


From the list of all the subject matter contents, we level

subject matter content. Then we can obtain the structure them into a decomposition level and tag them with of competence as in a figure 5 .

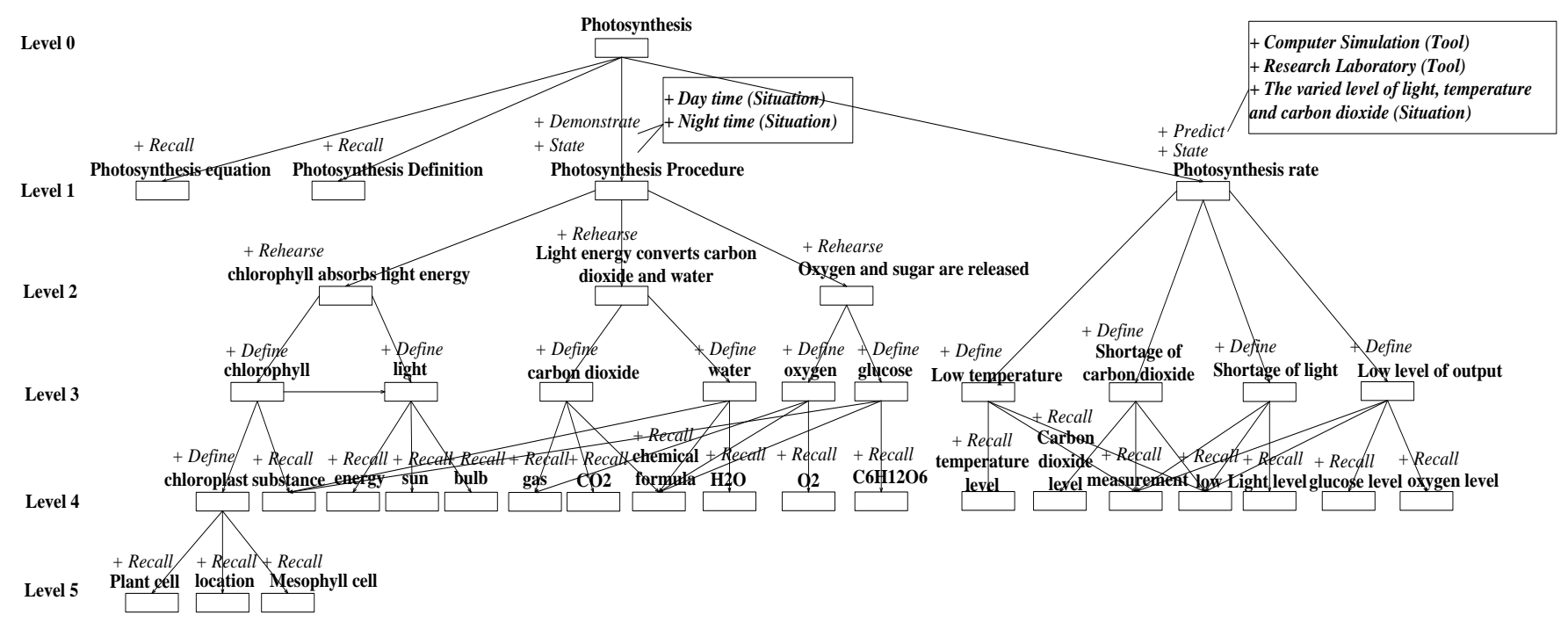

Figure 5. Competence Structure of Photosynthesis Knowledge Domain of Key Stage 4

The relationship between nodes of competences is represented as a parent-child relationship. It is similar to the previously developed competence structure. The described process of constructing a competence structure will help the developer understand the idea of transforming the course intended learning outcome into a competence structure.

\section{Mapped XML Schema}

There are some key points in representing a competence structure into an XML format. For example, XML enables us to focus on the definition of shared vocabularies for exchanging information and it easily reuses the content in other applications [12]. In this section, we describe the proposed class diagram which is considered from a competence structure and its corresponding XML schema.

\subsection{Class Diagram}

A class diagram in figure 6 represents all the main objectives in a competence structure such as, representation of intended learning outcome, different types of subject matter content (including its components) and representation of competence.

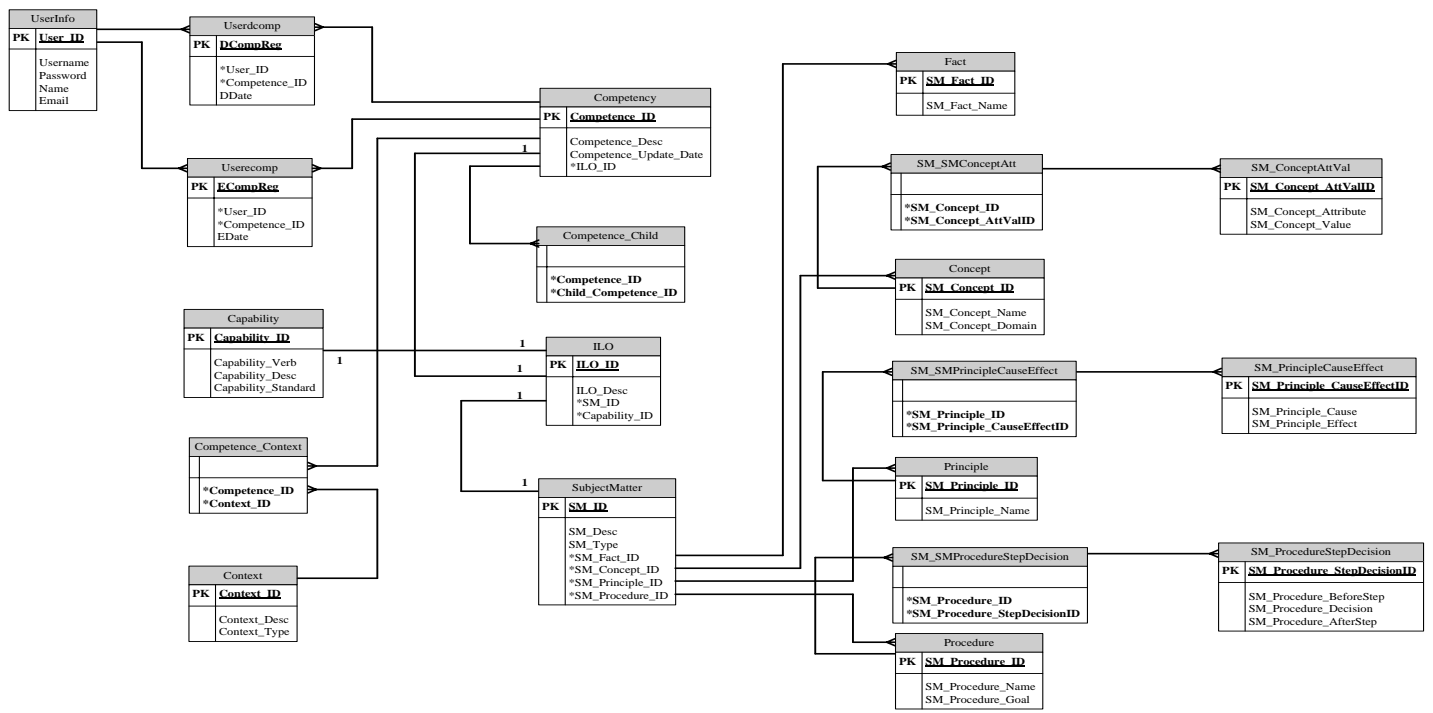

Figure 6. Class Diagram of Competence Structure 


\subsection{Mapping XML Schema}

XML-Schema defines the terms, relationships and constraints required to support communication in a particular application domain [13]. All schemas provide some degree of definition and documentation for an XML vocabulary. The definitions are useful both to system integration specialists (who are writing applications that process document instances of the vocabulary) and to web application specialists (who are developing stylesheets for transforming and presenting the XML content).

There are several reasons to create XML schema [13]:

- Defining and documenting the vocabulary for all users

- Validating documents when using XML parsers

- Providing structural guidance for content providers using XML authoring tools

- Providing default attribute values, enumerated lists and identifier declarations

- Defining new application or domain specific data types

For the competence based system it is essential to design an XML-schema since it represents a common framework for abstracting information of a competence structure. This XML-schema can be reusable for any knowledge domains of subject matter content. Figure 7 represent the structure of XML schema for a competence structure. The design of this schema is based upon the purposed class diagram in the previous sub-section.

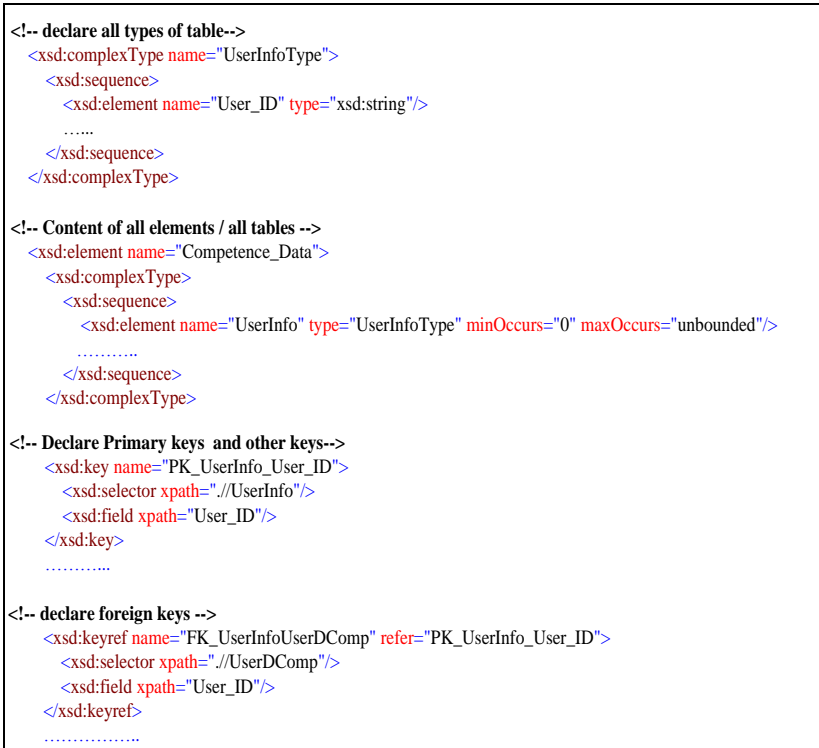

Figure 7. Mapped XML Schema of Competence Structure

\section{Implementation}

This section mainly gives the first stage of implementation. At this point the system was designed from a competence structure of H.C.F knowledge domain. For the first prototype, it allows a learner chooses his/her desired and existing competences from the boxes provided within knowledge domains (factor, common factor and highest common factor). The lists of desired and existing competences are obtained from DAG competence structure (figure 3 ) as we can see in figures 8 and 9. A list of existing competences contains child or children (including children/child of child) of a chosen desired competence. The system generates the search terms from existing and desired competence including the search results from Google search. Figure 10 shows the page on which the learner is required to choose his/her learning path. The list of learning paths is generated from chosen competences. After the learner chooses the learning path, the page suggesting study materials will be shown to him/her as shown in a figure 11. User interfaces are designed and coded with Html and PHP programming language.

\begin{tabular}{l} 
Choose Desired Competence \\
\hline Consider a sample of desired competence \\
By the end of this course, you (a learner) wish to be able to "calculate area of \\
triangle". Then a desired competence is "to calculate area of triangle" \\
*Desired competence indicates "what you would like to learn or achieve" \\
Hence, please choose your desired competence from the provided list. \\
0 evaluate highest common factor \\
0 evaluate common factor \\
evaluate factor \\
calculate factor \\
calculate common factor \\
calculate highest common factor \\
define highest common factor \\
define common factor \\
After you have chosen your desired competence, please click 'Next" below to \\
choose your existing competence.
\end{tabular}

Figure 8. A Page within a System on which the Learner chooses a desired competence

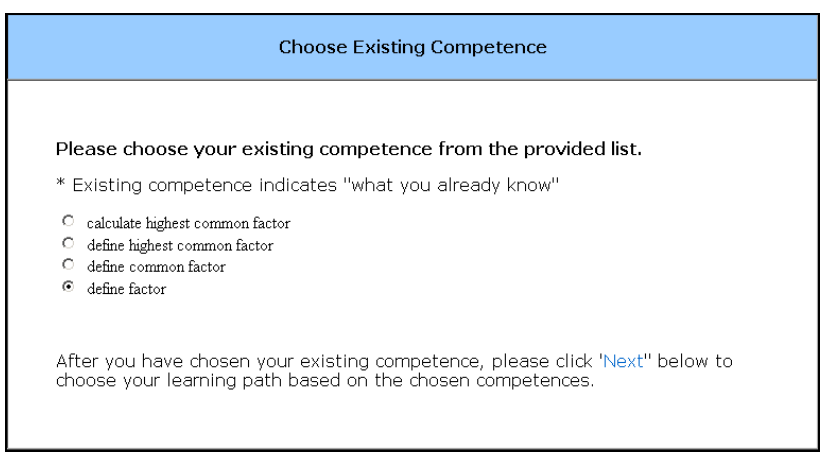

Figure 9. A Page within a System on which the Learner chooses an existing competence 


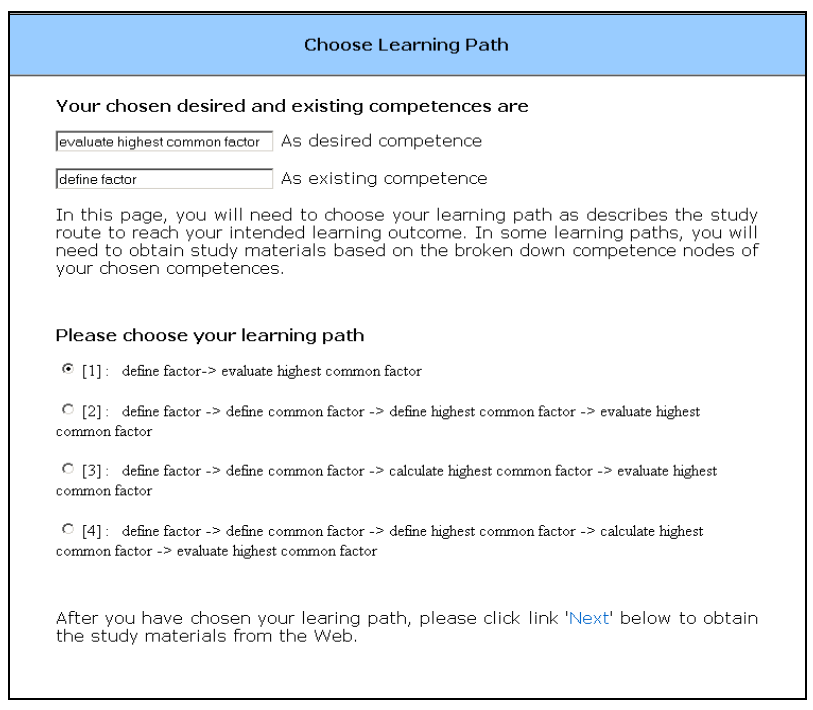

Figure 10. A Page Showing Different Learning Paths from Learner's Competences

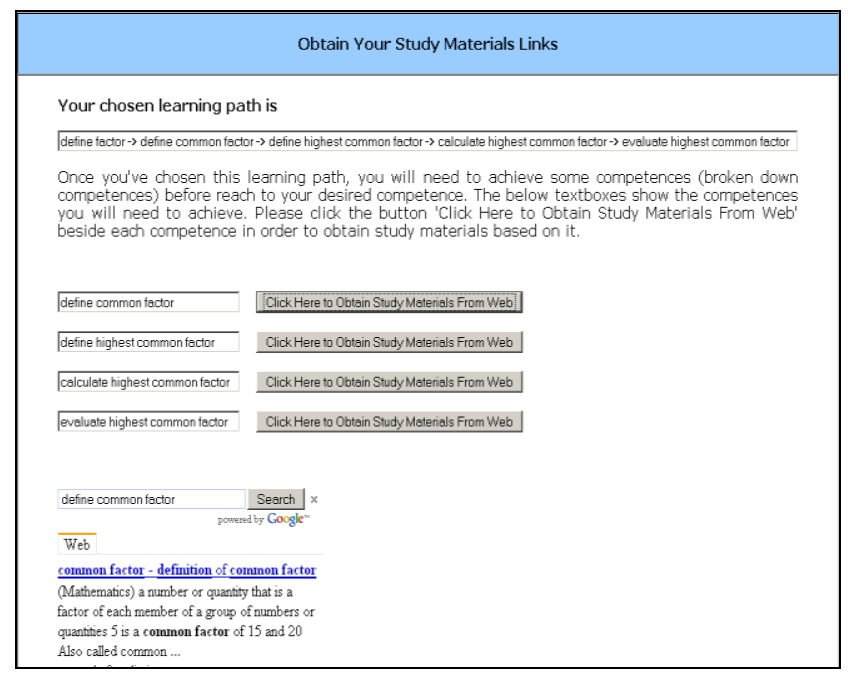

Figure 11. A Page within a System on which the Learner obtains Study materials from the Web

\section{Benefits of XML Database}

There are some advantages of using XML to represent the structure of competence. Firstly, the structure of competence is designed based on the intended learning outcomes of particular subject domain; normally this information is represented as the text file. The benefit of $\mathrm{XML}$ is that it is understandable by machine and human. Hence anyone can easily modify the content of XML file. It is easier for developers to locate and fix errors. Secondly, is the reusability issue; the content of competence structure can be changed for future use based on different knowledge domains. This allows the developers to reuse the XML file again. Thirdly, XML gives the flexibility of the language which allows the creation of custom data structures and organizational system. Hence the design of an XML file of competence structure becomes easy and the cost is inexpensive. Fourthly, is the advantage of XML over traditional database (RDBMs). XML structures data like a tree, while traditional database are all two-dimensional and rely on relations to describe data that does not fit in the structure [14].

\subsection{XML VS. Relational Database}

A previous implementation was developed with a relational database by using MySQL. However using $\mathrm{XML}$ to represent a competence structure is relatively better than using a relational database. There are some important criteria for comparison between XML and relational database (i.e. MySQL) based on the approach of designing structure of competence:

- Easiness: Using XML format it is easy to run filters and queries. In addition the formatted XML results do not need to be converted back to HTML. XML is more readable to both human and machine.

- Extensible: XML is extensible which allow it to extend the XML data structure [15]. Also XML itself is being extended with several additional standards that add styles, linking, and referencing ability to the core XML set of capabilities [12].

- Browser Accessibility: XML can be accessible via the browser.

- Conversion cost: XML is more efficient in terms of conversion cost [14].

- Reusability: We can reuse the XML file without any migration problem. XML content is easily reused in other applications or for other application [12].

- Modifiable: Both types of databases are modifiable but XML is more user-friendly modifiable.

- Semantic Approach: Basically W3C's Resource Description Framework (RDF) is an XML text format that supports resource description and metadata applications. With agreement on the meaning within RDF in order to communicate effectively, this can make the Web a little bit more into a Semantic Web [16].

From the discussion on the criteria between XML and relational database we can see that the consideration of $\mathrm{XML}$ in designing a competence structure is better than the consideration of it based on a relational database. There are some key criteria which we considered in this research work, for example extensible, reusability, semantic approach and explicit relationship. The first point is about extensibility and flexibility, these are to enable the XML schema of competence structure to be more extensible and flexible when we would like to change the information related to the competence structure, for example a type of context. The second point is a reusability issue; we can reuse or modify the XML 
file with different competence structures of various knowledge domains. The third issue is a semantic approach; this is significant since there are advantages to consideration of this approach with the design of competence structure, for example machine processable and the agreement of defining terms within a competence structure. In addition the approach of a semantic web is related with other issues for example, reusability and modifiability. The last issue is an explicit relationship, as stated previously, the defined elements and attributes in an XML file can be explicitly indicated as the relationships of competences within competence structure.

\subsection{Discussion on XML with Other Types of Structure}

Apart from a comparison between the uses of XML and relational databases for representing a competence structure, it is also important to consider other types of structures which could represent a competence structure for example, JSON and RDF.

JSON is a lightweight text-based open standard designed for human-readable data interchange [17]. JSON structure is derived from the JavaScript programming language for representing simple data structure and associated arrays called objects. The comparison points between XML and JSON are as follows:

- While both the JSON and XML forms can carry the same data, XML conveys semantic control/meaning, since XML needs an XSD describing the data obtained in the physical XML. In other words, XML is built for giving semantic meaning within documents while JSON is built for data structures [18].

- In general, XML syntax is much longer than JSON because XML attributes are more restricted than text elements in the set of characters they accept [19]. However XML encoding may be shorter than the equivalent JSON encoding if the XML document uses attributes rather than elements [19].

- JSON does not offer a way for unifying objects and ordered (number-indexed) arrays.

- XML lacks an explicit mechanism for representing large binary data types.

- JSON lacks explicit references, for example, extension in XML (XLink, XPointer).

RDF is a resource which is any web pages that can be identified with a URI. It is based upon the idea of making statements about resources. As XML is only a surface syntax for structured documents and imposes no semantic constraints on the meaning of these documents. By comparing RDF and XML based on a semantic web approach, RDF should be better than XML since we can parse the set of triples and then we can use the ones we want and ignore the one we do not understand [20]. The reason is we can create a data model for objects (or resources) and relations among them including providing a simple semantic for the data model which can be represented in XML syntax [20].

By considering a comparison of RDF with JSON based on a semantic approach, there are some advantages of RDF over JSON, for example we can make statements of equality between relations and things in RDF and there does not seem to be a notion of a namespace in JSON. In addition we cannot split data into something in pieces and put the information about those in URI since JSON does not have a URI as a base type.

In conclusion RDF is better than XML based on a semantic approach. However this research aims to propose a competence-based system for suggesting study material links based upon a learner's competences. There is a process within a system which is considered based on the structure of competence. The competence structure is modifiable and depends upon different knowledge domains of subject matter content. Hence the semantic approach is important for this research. Currently we purpose the XML structure of competence which can be modifiable based on different knowledge domain. RDF of competence structure can be considered in future work. We can implement the relations between nodes within a representation of subject matter with the defined relations in RDF. The focused part of this research is to get the keywords from learner's competences (capability+ subject matter + context) and we assume that the relations between nodes of competences will not affect the search results from a search engine. Hence the structure of competence in XML form is sufficient for generating the keywords from the learner's competences.

\section{Conclusion and Future Work}

In this paper we propose a design of a competence-based system for self-study and the process of transforming a course's intended learning outcomes into a form of XML schema for representing a competence structure. A major intended benefit of our system is its ability to provide study materials from the Web to learners in order that they can achieve their desired competences. Another concern is a competence structure which is designed based upon a course intended learning outcome. We consider an XML schema for representing a structure of competence since there are some advantages of reusability and modifiability. Hence this will allow the developers to easily modify the structure of competence based on different knowledge domain.

The future works will include the implementation of our system based on a new design of competence structure (photosynthesis knowledge domain). We tend to conduct the experiments to find out whether there are significant differences on the mean rating between all generated learning paths and also to find out the efficiency of the overall system. In addition, the experiment will also involve a study to find out whether there are significant differences on the mean rating 
between our competence-based system and other Webbased systems (could be a traditional search system).

\section{References}

[1] HR-XML, "Competencies (Measurable Characteristics Recommendation)," in $H R-X M L$ Consortium, C. Allen, Ed. USA: HR-XML Consortium, 2004.

[2] A. Stoof, R. Martens, and J. van Merriënboer, "Web-based support for constructing competence maps: design and formative evaluation," Educational Technology Research and Development, vol. 55, pp. 347-368, 2007.

[3] M. Eraut, Developing Professional Knowledge and Competence (Paperback). London: Falmer Press, 1994.

[4] IMS RDCEO, "IMS Reusable Definition of Competency or Educational Objective Information Model," 2002.

[5] HR-XML, "Competencies (Measurable Characteristics Recommendation)," in $H R-X M L$ Consortium, C. Allen, Ed. USA: HR-XML Consortium, 2004.

[6] D. Sampson and D. Fytros, "Competence Models in Technology-Enhanced Competence-Based Learning," in Handbook on Information Technologies for Education and Training, 2008, pp. 155-177.

[7] O. Sitthisak, L. Gilbert, and H. Davis, "TRANSFORMING A COMPETENCY MODEL TO ASSESSMENT ITEMS " in 4th International Conference on Web Information Systems and Technologies (WEBIST) Funchal, Madeira - Portugal, 2008.

[8] L. Gilbert and V. Gale, Principles of E-learning Systems Engineering. Oxford, UK: Chandos Publishing, 2008.

[9] D. Laurillard, Rethinking university teaching : a framework for the effective use of educational technology 2nd ed. London: Routledge, 1993.

[10] The Assessment and Qualifications Alliance, "GCSE Specification - Biology," 2010.

[11] M. D. Merrill, Instructional Design Theory. New Jersey: Educational Technology Publication, 1994.

[12] S. St.Laurent, "Why XML?," 1998.

[13] D. Carlson, Modeling XML applications with UML: practical e-Business applications Addison-Wesley Professional, 2001.

[14] D. Obasanjo, "An exploration of XML in database management systems," 2001.

[15] V. Viarengo, "What about XML Extensibility?," 2001.

[16] B. Bos, "XML in 10 points," 2001.

[17] JSON official website, "Introducing JSON," 2010.

[18] J. Cormack, "JSON vs XML," 2010.
[19] N. Nurseitov, M. Paulson, R. Reynolds, and C. Izurieta, "Comparison of JSON and XML Data Interchange Formats: A Case Study," CAINE, 2009.

[20] T. Berners-Lee, "Why RDF model is different from the XML model ", 1998. 